\title{
Neuroendoscopic minimally invasive surgery and small bone window craniotomy hematoma clearance in the treatment of hypertensive cerebral hemorrhage
}

\author{
Chengjia Gui ${ }^{1}$, Yikuan $\mathrm{Gao}^{2}$, Dan $\mathrm{Hu}^{3}$, Xinyu Yang ${ }^{4}$
}

\begin{abstract}
Objective: To analyze the effects of neuroendoscopic minimally invasive surgery and small bone window craniotomy hematoma clearance through comparing clinical indicators of the two operation modes and to provide a reference for selection of proper minimally invasive surgery.

Methods: One hundred and twenty-six patients with hypertensive cerebral hemorrhage who received diagnosis and treatment in our hospital between December 2015 and December 2017 were selected and grouped into an observation group $(n=63)$ and a control group $(n=63)$ using random number table. Patients in the observation group were treated by neuroendoscopic surgery, while patients in the control group were treated by small bone window craniotomy. The surgical condition, clinical effect and prognosis of the two groups were analyzed and compared.

Results: Patients in the observation group completed surgery in a shorter time and bled less during operation compared to the control group, and the hematoma clearance rate of the observation group was obviously higher than that of the control group; the differences had statistical significance $(P<0.05)$. The nerve deficiency scale (NDS) scores of the two groups at the postoperative $3^{\text {rd }}$ month were lower than those before surgery $(P<0.05)$, and the activity of daily life $(A D L)$ score at the postoperative $3^{\text {rd }}$ month was higher than that before surgery $(\mathrm{P}<0.05)$. The observation group had lower NDS score and higher ADL score compared to the control group, and the differences had statistical significance $(P<0.05)$. The incidence of complications of the observation group was lower than that of the control group after surgery, and the rate of favourable prognosis of the observation group was higher than that of the control group at the postoperative $3^{\text {rd }}$ month $(\mathrm{P}<0.05)$.

Conclusion: Neuroendoscopic surgery is more effective and safe, causes less bleeding and has better prognosis and nerve function recovery compared to small bone window craniotomy in the treatment of hypertensive cerebral hemorrhage.
\end{abstract}

KEY WORDS: Hypertension, Cerebral hemorrhage, Neuroendoscopy, Small bone window craniotomy.

How to cite this:

doi: https://doi.org/10.12669/pjms.35.2.463

Gui C, Gao Y, Hu D, Yang X. Neuroendoscopic minimally invasive surgery and small bone window craniotomy hematoma clearance in the treatment of hypertensive cerebral hemorrhage. Pak J Med Sci. 2019;35(2):377-382. doi: https://doi.org/10.12669/pjms.35.2.463

This is an Open Access article distributed under the terms of the Creative Commons Attribution License (http://creativecommons.org/licenses/by/3.0), which permits unrestricted use, distribution, and reproduction in any medium, provided the original work is properly cited.

Correspondence:

Xinyu Yang,

Department of Neurosurgery,

Tianjin Medical University General Hospital,

Tianjin 300052, China.

E-mail: yangxinyubd@163.com

* Received for Publication:

* $1^{\text {st }}$ Revision Received:

* $\quad 2^{\text {nd }}$ Revision Received:

* Final Revision Accepted:
July 19, 2018

November 12, 2018

January 28, 2019

January 30, 2019

\section{INTRODUCTION}

Hypertensive intracerebral hemorrhage $(\mathrm{HICH})$ is one of the common acute cerebrovascular diseases and also one of the common types of cerebral hemorrhage. Now it has been an important cause of death, ${ }^{1}$ which has severely threatened the physical and psychological health of human. Guidelines for the Management of Spontaneous 
Intracerebral Hemorrhage released by American Heart Association in 2015 pointed out that about $10 \sim 15 \%$ of patients who were attacked by stroke for the first time had $\mathrm{HICH}$, the mortality rate was about $50 \%$, about half of the dead died within 24 hours after onset, ${ }^{2}$ and only $28 \% \sim 35 \%$ of the survivors had independent living ability. ${ }^{3}$ Someone in China found that the incidence of $\mathrm{HICH}$ in China was $0.72 / 10000 \sim 1.15 / 10000$, most of them aged over 55 years, and the incidence among young people became increasingly higher. ${ }^{4,5}$ There are many patients with $\mathrm{HICH}$ in China because of the large population base, which brings huge burdens to the family members of the patients and the society and severely affects the living level of people. Therefore how to select effective diagnostic and treatment methods for $\mathrm{HICH}$, improve the survival rate and enhance living quality of patients in the late stage has been the dilemma.

The key of treating $\mathrm{HICH}$ is eliminating space occupying effect, removing hematoma, decreasing intracranial pressure, repairing injured neurons, preventing secondary pathological injury and promoting prognosis as soon as possible. ${ }^{6}$ Patients with $\mathrm{HICH}$ who have a small amount of blood loss can get a good outcome after being treated by drugs. Patients who bleed more than $30 \mathrm{~mL}$ are usually treated by surgery. ${ }^{7}$ Gregson et al. found that surgical treatment was more effective than conservative treatment for patients with cerebral hemorrhage who underwent early surgery within eight hours after hemorrhage, ${ }^{8}$ had $20 \sim 50 \mathrm{~mL}$ of blood loss, had 9 12 points of Glasgow Coma Scale (GCS) score, and aged 50 69 years. Small bone window craniotomy is a traditional surgical method for treating $\mathrm{HICH}$, and its effect has been widely recognized. But it has shortcomings of large trauma and multiple postoperative complications. ${ }^{9,10}$ With the development of minimally invasive technology, endoscopic neurosurgery has been extensively performed in clinics for its advantages of less pain, fast recovery and low incidence of postoperative complications. It has been the main surgical method for treating $\mathrm{HICH} .{ }^{11,12}$

Through comparing the effects of endoscopic neurosurgery, stereotactic treatment and craniotomy for 30 patients with cerebral hemorrhage in basal ganglia, Cho et al. found that endoscopic neurosurgery had the highest hematoma clearance rate and the shortest operation time. ${ }^{13}$ However, the treatment criteria have not been unified though there are many studies concerning neuro endoscopy. The selection of surgical method, therapeutic effect and prognosis of $\mathrm{HICH}$ are still being explored. In this study, the effects of neuro endoscopic minimally invasive surgery and small bone window craniotomy were compared. This study can guide clinical physicians to select reasonable treatment schemes for $\mathrm{HICH}$ and figure out the effectiveness of endoscopic neurosurgery in the treatment of $\mathrm{HICH}$.

\section{METHODS}

One hundred and twenty-six patients with $\mathrm{HICH}$ who received diagnosis and treatment in our hospital between December 2015 and December 2017 were enrolled. All of them satisfied the diagnostic criteria formulated by WHO/ISH in $1999 .{ }^{14}$ They were admitted to the hospital within 12 hours after onset and were definitely diagnosed by head computed tomography (CT). The patients had 30 60 mL of supratentorial hemorrhage, 5 13 points of GCS score, grade II IV consciousness disorder, and history of hypertension. Patients who had more than $60 \mathrm{~mL}$ of blood loss, need undergoing decompressive craniectomy because of severe cerebral edema, had cerebellar hemorrhage, brainstem hemorrhage, craniocerebral injury, arterial aneurysm or arteriovenous malformation, or have took anticoagulant drugs for a long term were excluded. The patients were divided into an observation group and a control group using random number table, 63 cases in each group. In the observation group, there were 39 males and 24 females, and they aged 40 71 years (54.02 \pm 3.74 years) and had a hypertension history of $4 \sim 21$ years (average $8.21 \pm 1.06$ years), 35 73 $\mathrm{mL}$ of hematoma (average 52.39 \pm 3.64 ) and 4 12 points of GCS score (average 6.01 \pm 0.64 points). In the control group, there were 36 males and 27 females, and they aged 37 71 years (average 52.33 \pm 3.41 years) and had a hypertension history of 3 22 years (average 7.38 \pm 0.75 years), $30 \sim 69 \mathrm{~mL}$ of hematoma and $3 \sim 13$ points of GCS score (average $6.35 \pm 0.71$ points). The differences of gender and age between the two groups had no statistical significance $(\mathrm{P}>0.05)$; hence the results were comparable. This study has been approved by the ethical committee of our hospital, and all the patients signed informed consent.

Patients in the observation group underwent endoscopic neurosurgery. The position of hematoma was determined using head CT. The site which was the nearest to the largest hematoma and away from important nerves, blood vessels and brain function region was taken as the surgical approach. After general anesthesia, a $5 \mathrm{~cm}$ incision 
Treatment of hypertensive cerebral hemorrhage

Table-I: Surgical indexes between the two groups (mean \pm SD).

\begin{tabular}{lccc}
\hline Group & Duration of surgery $(h)$ & Intraoperative blood loss $(\mathrm{mL})$ & Hematoma clearance rate $(\%)$ \\
\hline Observation group & $1.69 \pm 0.87$ & $35.61 \pm 13.52$ & $92.31 \pm 8.52$ \\
Control group & $3.69 \pm 1.34$ & $277.14 \pm 101.33$ & $78.23 \pm 7.74$ \\
$\mathrm{t}$ & 8.497 & 23.269 & 8.574 \\
$\mathrm{p}$ & $<0.05$ & $<0.05$ & $<0.05$ \\
\hline
\end{tabular}

was made. The scalp tissue was retracted to fully expose the cranium. A bone hole whose diameter was $2 \mathrm{~cm}$ was drilled. The endocranium was incised. A neuroendoscopy set (STORZ, Germany) was punctured into the hematoma cavity. After the inner core was removed, the neuroendoscope was inserted. The hematoma was removed under the neuro endoscopy. Electrocoagulation could be used if there was bleeding. Drainage tubes were inserted after surgery. Finally the incision was sutured.

Patients in the control group underwent small bone window craniotomy. After general anesthesia, the position of hematoma was determined according to the results of head CT. A $6 \mathrm{~cm}$ incision was cut on the scalp. Then a hole was drilled. The size of small bone window was about $4.0 \mathrm{~cm} \times 5.0 \mathrm{~cm}$. The endocranium was incised. Then the position of hematoma was determined using brain puncture. The cerebral cortex was incised. After hematoma elimination, epidural drainage tube was inserted. Then the cranium was closed. After surgery, patients in both groups were given treatment to resist infection, maintain water and electrolytes balance and reduce intracranial pressure.

The intraoperative blood loss, duration of surgery, hematoma clearance rate and incidence of postoperative complications were compared and analyzed. The formula of hematoma clearance rate was: hematoma clearance rate $=$ (preoperative hematoma volume under CT - hematoma volume under CT within 24 hour after surgery)/ preoperative hematoma volume under $\mathrm{CT}$. All the patients were followed up for three months. The degree of neurological deficit of the patients was evaluated using clinical nerve deficiency scale (NDS) before surgery and at the postoperative $3^{\text {rd }}$ month, and the score was 0 point $\sim 45$ points. The high the
NDS score, the severer the neurologic impairment. The ability of daily living was evaluated using activity of daily living scale (ADL) before surgery and at the postoperative $3^{\text {rd }}$ month. Higher score of ADL indicated better activity of daily living. Good recovery was given five points; mild disability was given four points; severe disability was given 3 points; vegetative state was given two points; death was given one point. Favourable prognosis was given 4 or 5 points, and poor prognosis was given $1 \sim 3$ points.

Statistical method: Data were processed using SPSS ver. 21.0. Measurement data were expressed as mean \pm standard deviation (SD) and tested by $\mathrm{t}$ test. Categorical data were expressed as rate (\%) and tested by Chi-square test. Difference was considered statistically significant if $\mathrm{P}<0.05$.

\section{RESULTS}

The duration of surgery and intraoperative blood loss of the observation group were less than those of the control group, and the hematoma clearance rate of the observation group was obviously higher than that of the control group $(\mathrm{P}<0.05$, Table-I).

The differences of ADL and NDS scores between the two groups had statistical significance before treatment $(\mathrm{P}>0.05)$. The NDS score of the two groups at the postoperative $3^{\text {rd }}$ month was lower than that before treatment, and the ADL score at the postoperative $3^{\text {rd }}$ month was higher than that before treatment $(\mathrm{P}<0.05)$. The NDS score of the observation group was obviously lower than that of the control group, and the ADL score of the observation group was obviously higher than that of the control group, as shown in Table-II.

In the control group, there were four cases of intracranial infection and five cases of pulmonary

Table-II: ADL and NDS score between the two groups (mean \pm SD).

\begin{tabular}{llcl}
\hline Group & Time & ADL score & NDS score \\
\hline Observation group & Before treatment & $29.87 \pm 3.73$ & $41.26 \pm 5.67$ \\
& Postoperative 3rd month & $66.86 \pm 5.87^{*} \#$ & $22.12 \pm 3.01^{*} \#$ \\
Control group & Before treatment & $30.01 \pm 3.82$ & $40.79 \pm 5.74$ \\
& Postoperative 3rd month & $44.79 \pm 4.52^{*}$ & $31.72 \pm 3.19^{*}$ \\
\hline
\end{tabular}

Note: * indicated $\mathrm{P}<0.05$ compared to before treatment; \# indicated $\mathrm{P}<0.05$ compared to the control group. 
Table-III: Comparison of prognosis between the two groups at the postoperative 3rd month [n(\%)].

\begin{tabular}{lcccccc}
\hline Group & 1 point & 2 points & 3 points & 4 points & 5 points & Favourable prognosis \\
\hline Observation group & $4(6.35)$ & $7(11.11)$ & $13(20.34)$ & $15(23.81)$ & $24(38.09)$ & $39(61.90) \#$ \\
Control group & $9(14.29)$ & $11(17.46)$ & $17(26.98)$ & $12(19.05)$ & $14(22.22)$ & $26(41.27)$ \\
\hline
\end{tabular}

Note: \# indicated $\mathrm{P}<0.05$ compared to the control group.



Fig.1: Head CT of a 58-year-old female patient in the observation group before and after surgery (A: preoperative; B: postoperative).

infection; the incidence of complications was $14.29 \%$. In the observation group, there was only one case of pulmonary infection; the incidence of complications was $1.59 \%$. The incidence of complications of the observation group was significantly lower than that of the control group $\left(\mathrm{X}^{2}=4.913, \mathrm{P}<0.05\right)$.

The rate of favorable prognosis of the observation group was $61.90 \%$ at the postoperative $3^{\text {rd }}$ month, which was significantly higher than that of the control group $(41.27 \%)\left(\mathrm{X}^{2}=4.174, \mathrm{P}<0.05\right.$, Table-III). The typical cases are shown in Fig.1 and 2.

\section{DISCUSSION}

$\mathrm{HICH}$ refers to a hemorrhagic disease which occurs secondary to hypertension, which has a high incidence in China. $\mathrm{HICH}$ can produce primary or secondary damages to brain tissues. ${ }^{15}$ Primary damage mainly manifests as cerebral hernia induced by the compression on brain tissues because of the space occupying effect of hematoma. It may threaten the lives of patients in severe cases. Secondary damage which is induced by the substances released by brain cell damages and hemolysis can further induce edema and dysfunction of brain tissues, aggravating disease condition. ${ }^{16}$ As the occupied effect induced by acute intracranial hematoma and pathological damage induced by bleeding is the main cause of disability and death, intracranial hematoma should be cleaned by clinical treatment as soon as possible

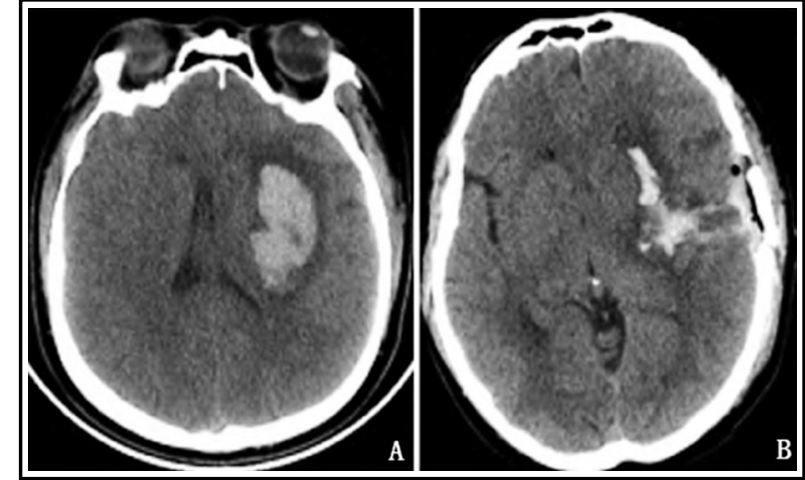

Fig.2: Head CT of a 53-year-old female patient in the control group before and after surgery

(A: preoperative; B: postoperative).

to relieve the occupied effect of hematoma, release brain tissue oppressed by hematoma, alleviate encephaledema, reduce intracranial pressure, and improve the prognosis of patients. ${ }^{2,17}$

There are many operation methods for treating $\mathrm{HICH}$ currently, but the standard method is still controversial, and every surgical procedures has indications and complications. In the application of craniotomy evacuation of hematoma, operation can be performed under direction vision, hematoma can be thoroughly removed, the hemostatic effect is definite. Moreover whether decompressive craniectomy is needed or not can be determined according to intraoperative intracranial pressure. Its complications include large trauma, severe hemorrhage, long duration of surgery and poor prognosis. ${ }^{18}$ Under the influence of the general tendency of minimally invasive surgery, neuroendoscopy has gained substantial development in the field of neurosurgery. ${ }^{19}$ Neuroendoscopy has special advantages in the treatment of $\mathrm{HICH}$, including removal of hematoma under direct vision, multi-angle rotation of endoscope, small trauma, short duration of operation and small blood loss. Under direct vision, the good lighting can help fully expose lesions and reduce damages to neurons and important blood vessels. The multi-angle rotation of endoscope can help thoroughly remove hematoma at different positions. Someone ${ }^{20}$ pointed out 
that the hematoma clearance rate of endoscopic neurosurgery was between $86 \%$ and $100 \%$ and that endoscopic neurosurgery had advantages of small trauma, short operation time and small bleeding volume. The results of this study demonstrated that the observation group had shorter operation time, smaller intraoperative bleeding volume and significantly higher hematoma clearance rate compared to the control group, which was consistent with the aforementioned mechanism and previous research results.

The long duration of craniotomy has large damages to the whole body and brain of patients. Therefore surgery associated complications including infection, organ failure, rehaemorrhagia and thrombogenesis are prone to occur in the respiratory system, urinary system, nervous system and digestive system after craniotomy. ${ }^{21}$ Though small bone window craniotomy can effectively reduce the incidences of some complications, the incidence of surgery associated complications is still up to $40 \%$, and some patients have died of the complications. ${ }^{22}$ Neuroendoscopic hematoma evacuation can also induce similar complications in the treatment of cerebral hemorrhage, but the incidence of complications is much lower. It is found that the incidence of complications of neuroendoscopic hematoma evacuation was 3.3\%, much lower than that of stereotactic intracranial hematoma removal $(10 \%)$ and craniotomy $(16.6 \%)$ in the treatment of cerebral hemorrhage in basal ganglia $(\mathrm{P}<0.05) \cdot{ }^{13}$ The results of this study demonstrated that the incidence of postoperative complications of the observation group was significantly lower than that of the control group, which was consistent with the aforementioned research result.

The neurological recovery, activity of daily living and long-term curative effect of the patients were evaluated using NDS, ADL and GCS scores respectively at the postoperative $3^{\text {rd }}$ month. The results demonstrated that the NDS score of the observation group was significantly lower than that of the control group, the ADL score of the observation group was significantly higher than that of the control group, and the rate of favourable prognosis of the observation group was significantly higher than that of the control group, suggesting that endoscopic neurosurgery had special advantage in improving the neurological function, living ability and prognosis of patients with $\mathrm{HICH}$. The finding was consistent with the research results of Chan et al..$^{23}$ The main reason of the above result is the small trauma that endoscopic neurosurgery brought to patients, not only reflecting on the small damages to brain tissue, but also reflecting on the short duration of surgery, small blood loss and low tolerance to surgery. Therefore the patients recovered fast, had less complication, their hospital stay was short, and started functional exercise earlier.

\section{CONCLUSION}

Endoscopic neurosurgery has advantages of small trauma, mild blood loss, and short duration of surgery, high hematoma clearance rate, low incidence of complications, rapid recovery and favorable prognosis for patients with $\mathrm{HICH}$. With the constant development of science and technology and the accumulation of experience in using neuroendoscopy, neuroendoscopy is bound to play an increasingly important role in the field of neurosurgery and will be accepted by more and more physicians.

Acknowledgment: National Natural Science Foundation of China (81571144) and Natural Science Foundation of Tianjin City (16JCZDJC35700).

Declaration of interest: All authors declared there was no conflict interests involved.

\section{Grant Support \& Financial Disclosures: None.}

\section{REFERENCES}

1. Xu S, Pang Q, Lin Z, Zhang N. Effect of integrated traditional Chinese and Western medicine therapy for acute hypertensive intracerebral hemorrhage: A metaanalysis. Artif Cells Nanomed Biotechnol. 2016;28:1-6. doi: 10.1080/21691401.2016.1215327

2. Yang G, Shao G. Clinical effect of minimally invasive intracranial hematoma in treating hypertensive cerebral hemorrhage. Pak J Med Sci. 2016;32(3):677-681. doi: 10.12669/pjms.323.9533

3. Kang J, Huang Q, Liu Y. Advance in research on the genetic etiology of spontaneous intracerebral hemorrhage. Chin J Med Genet. 2016;33(5):702-707. doi: 10.3760/cma.j.is sn.1003-9406.2016.05.028

4. Qureshi AI, Palesch YY, Barsan WG, Hanley DF, Hsu CY, Martin RL, et al. Intensive blood-pressure lowering in patients with acute cerebral hemorrhage. N Engl J Med. 2016;375(11):1033-1043. doi: 10.1056/NEJMoa1603460

5. Jolink WM, Klijn CJ, Brouwers PJ, Kappelle LJ, Vaartjes I. Time trends in incidence, case fatality, and mortality of intracerebral hemorrhage. Neurol. 2015;85(15):1318-1324. doi: 10.1212/WNL.0000000000002015

6. Chi FL, Lang TC, Sun SJ, Tang XJ, Xu SY, Zheng HB, et al. Relationship between different surgical methods, hemorrhage position, hemorrhage volume, surgical timing, and treatment outcome of hypertensive intracerebral hemorrhage. World J Emerg Med. 2014;5(3):203-208. doi: 10.5847/wjem.j.1920-8642.2014.03.008 
7. Han FL, Zhang GL, Wu SG, Zhou ZW, Han XP. Efficacy of small bone window craniotomy and large bone flap craniotomy in the treatment of hypertensive cerebral hemorrhage. Prog Mod Biomed. 2016;16(33):6542-6545. doi: 10.13241/j.cnki.pmb.2016.33.038

8. Gregson BA, Broderick JP, Auer LM, Batjer H, Chen $X C$, Juvela $S$, et al. Individual patient data subgroup meta-analysis of surgery for spontaneous supratentorial intracerebral hemorrhage. Stroke. 2012; 43(6): 1496-1504. doi: 10.1161/STROKEAHA.111.640284

9. Laiwattana D, Sangsawang B, Sangsawang N. Primary multiple simultaneous intracerebral hemorrhages between 1950 and 2013: analysis of data on age, sex and outcome. Cerebrovasc Dis Extra. 2014;4(2):102-114. doi: $10.1159 / 000362111$

10. Liu S. Comparison of curative effects of small bone window hematoma evacuation and craniotomy accompanied by cranioplasty. Contemp Med. 2014;(5):105-106. doi: 10.3969/j issn.1009-4393.2014.05.075

11. Jia Z, Mohammed W, Qiu Y, Hong X, Shi H. Hypertension increases the risk of cerebral microbleed in the territory of posterior cerebral artery: a study of the association of microbleeds categorized on a basis of vascular territories and cardiovascular risk factors. J Stroke Cerebrovasc Dis. 2014;23(1):e5-11. doi: 10.1016/j. jstrokecerebrovasdis.2012.12.016

12. Hwang BY, Bruce SS, Appelboom G, Piazza MA, Carpenter AM, Gigante PR, et al. Evaluation of intraventricular hemorrhage assessment methods for predicting outcome following intracerebral hemorrhage. Neurosurg. 2012;116(1):185-192. doi: 10.3171/2011.9.JNS10850

13. Cho DY, Chen CC, Chang CS, Lee WY, Tso M. Endoscopic surgery for spontaneous basal ganglia hemorrhage: comparing endoscopic surgery, stereotactic aspiration, and craniotomy in noncomatose patients. Surg Neurol. 2006;65(6):547-556.

14. Wu QF, Wang BY, Zheng X, Wang DD, Geng WF, Liu BX, et al. Clinical analysis on punch drainage and lateral ventricle drainage in the treatment of 27 cases of hematodialysis and cerebral hemorrhage. Chin J Neuromed. 2016;29(10):39-40.

15. Passos GF, Kilday K, Gillen DL, Cribbs DH, Vasilevko V. Experimental hypertension increases spontaneous intracerebral hemorrhages in a mouse model of cerebral amyloidosis. J Cereb Blood Flow Metab. 2016;36(2):399-404 doi: $10.1177 / 0271678 \times 15606720$

16. Schlunk F, Greenberg SM. The Pathophysiology of intracerebral hemorrhage formation and expansion. transl stroke res. 2015; 6(04): 257-263. doi: 10.1007/ s12975-015-0410-1
17. Riveralara L, Murthy SB, Nekoovaghttak S, Ali H, McBee N, Dlugash $\mathrm{R}$, et al. Influence of bleeding pattern on ischemic lesions after spontaneous hypertensive intracerebral hemorrhage with intraventricular hemorrhage. Neurocrit Care 2018(Suppl 1):1-9.

18. Zhang HZ, Li YP, Yan ZC, Wang XD, She L, Wang XD, et al. Endoscopic evacuation of basal ganglia hemorrhage via keyhole approach using an adjustable cannula in comparison with craniotomy. Biomed Res Int. 2014;2014:898762. doi: $10.1155 / 2014 / 898762$

19. Wang WH, Hung YC, Hsu SP, Lin CF, Chen HH, Shih $\mathrm{YH}$, et al. Endoscopic hematoma evacuation in patients with spontaneous supratentorial intracerebral hemorrhage. J Chin Med Assoc. 2015;78(2):101-107. doi: 10.1016/j. jcma.2014.08.013

20. Wang ZF, Liu F, Liao DG, Zhang TY. Endoscopic surgery for hypertensive cerebral hemorrhage. J Cent South Univ. 2005; 30(4): 424-426.

21. Ding D. Role of surgical evacuation in the management of giant hypertensive intracerebral hemorrhage. Clin Neurol Neurosurg. 2013;115(12):2555-2556. doi: 10.1016/j. clineuro.2013.09.041

22. Zhang T, Zou HS, Chen WQ. A comparative study of therapeutic effects of small bone flap craniotomy and traditional craniotomy in patients with hypertensive cerebral hemorrhage. Chin J Neuromed. 2011;10(9):953-954. doi: 10.3760/cma.j.issn.1671-8925.2011.09.023

23. Chan E, Anderson CS, Wang X, Arima H, Saxena A, Moullaali TJ, et al. Significance of intraventricular hemorrhage in acute intracerebral hemorrhage: intensive blood pressure reduction in acute cerebral hemorrhage trial results. Stroke. 2015;46(3):653-658. doi: 10.1161/STROKEAHA.114.008470

\section{Authors' Contribution:}

CJG \& XYY: Study design, data collection and analysis.

YKG, DH \& XYY: Manuscript preparation, drafting and revising.

XYY: Review and final approval of manuscript.

Authors:
1. Chengjia Gui,
2. Yikuan Gao,
3. Dan Hu,
Department of Neurosurgery,
Central Hospital of Yongzhou,
Hunan, 425000, China.
Xinyu Yang,
Department of Neurosurgery,
Tianjin Medical University General Hospital,
Tianjin 300052, China.
1, 2: Department of Neurosurgery,
Tianjin Medical University General Hospital,
Tianjin 300052, China.
Department of Neurosurgery,
Central Hospital of Yongzhou,
Hunan, 425000, China.

